\title{
Symbolic Computation Applications in Power Engineering Education
}

\section{Dr. Radian G Belu, Southern University}

Dr. Radian Belu is Associate Professor within Electrical Engineering Department, Southern University, Baton, Rouge, USA. He is holding one PHD in power engineering and other one in physics. Before joining to Southern University Dr. Belu hold faculty, research and industry positions at universities and research institutes in Romania, Canada and United States. He also worked for several years in industry as project manager, senior engineer and consultant. He has taught and developed undergraduate and graduate courses in power electronics, power systems, renewable energy, smart grids, control, electric machines, instrumentation, radar and remote sensing, numerical methods, space and atmosphere physics, and applied physics. His research interests included power system stability, control and protection, renewable energy system analysis, assessment and design, smart microgrids, power electronics and electric machines for non-conventional energy conversion, remote sensing, wave and turbulence, numerical modeling, electromagnetic compatibility and engineering education. During his career Dr. Belu published ten book chapters, several papers in referred journals and in conference proceedings in his areas of the research interests. He has also been PI or Co-PI for various research projects United States and abroad in power systems analysis and protection, load and energy demand forecasting, renewable energy, microgrids, wave and turbulence, radar and remote sensing, instrumentation, atmosphere physics, electromagnetic compatibility, and engineering education.

\section{Prof. Lucian Ionel Cioca, Lucian Blaga University of Sibiu}

Lucian Ionel CIOCA received the M.Sc. in Machine Tools (1993) and B.Sc. in Occupational Safety, Health and Work Relations Management (2010). In 2002, he becomes Dr. Eng. (Ph.D degree) of Petrosani University, Romania and now he is professor at "Lucian Blaga" University of Sibiu - Romania, Faculty of Engineering, Department of Industrial Engineering and Management, Romania. His teaching subjects are Ergonomics, Management, Human Resources Management, Occupational Health and Safety Management, Production Systems Engineering. His research fields of interest are linked with the impact of the knowledge based society upon the social / human dynamics / evolution and the production systems. He regularly publishes and participates on international scientific conferences. Lucian Cioca is the Administrator of the LBUS Department of Consulting, Training and Lifelong Learning, Doctoral Advisor in Engineering and Management, Member of the National Council for Attestation of Academic Titles, Diplomas and Certificates, evaluator ARACIS (The Romanian Agency for Quality Assurance in Higher Education), and other (email: lucian.cioca@ulbsibiu).

\section{Dr. Richard Chiou, Drexel University (Eng. \& Eng. Tech.)}

Dr. Richard Chiou is Associate Professor within the Engineering Technology Department at Drexel University, Philadelphia, USA. He received his Ph.D. degree in the G.W. Woodruff School of Mechanical Engineering at Georgia Institute of Technology. His educational background is in manufacturing with an emphasis on mechatronics. In addition to his many years of industrial experience, he has taught many different engineering and technology courses at undergraduate and graduate levels. His tremendous research experience in manufacturing includes environmentally conscious manufacturing, Internet based robotics, and Web based quality. In the past years, he has been involved in sustainable manufacturing for maximizing energy and material recovery while minimizing environmental impact. 


\title{
Symbolic Computation Applications in Power Engineering Education
}

Radian Belu, Southern University, Baton Rouge. USA

Lucian Cioca, University "Lucian Blaga” of Sibiu, Romania

\begin{abstract}
The complexity of power system problems requires the use of modern, multiple and advanced computing tools and programs. Software applications in power system operation, analysis and management began several decades ago. As power systems have become increasingly complex and moving towards smart grids there are also critical needs to have available similar tools and programs for training power engineering students. An attractive way of teaching power engineering topics is the use of computer algebra systems. These interactive environments for computation, visualization, and modeling are used to analyze a wide variety of engineering and science problems. They are designed to obtain explicit symbolic solutions to engineering and scientific problems and have advanced graphic and visualization capabilities. Moreover, software packages designed to numerically solve problems, such as MATLAB now include symbolic capabilities, transforming them into symbolic-assisted numeric computational tools. This paper focuses on how such software packages can be used to improve power engineering education. The advantages and disadvantages of the use of symbolic computations in power engineering courses are also discussed. Lessons learned are included and feedback and suggestions from other educators are welcomed.
\end{abstract}

\section{Introduction, Power Engineering Education Issues and Challenges}

Excellence in engineering education comes from innovative teaching and effective instructional materials, requiring often changing the traditional way of delivering engineering courses. In the traditional teaching methods, lecturers offer course materials in a classroom where students listen, take notes, copy materials, execute homework and complete assignments. Quite often lecturers fail to transfer knowledge to students effectively despite personally having sound technical knowledge in the subject areas. Engineering educators have stressed the importance of presenting technical information in various learning styles and/or using advanced computer technologies. The aim of this paper is to examine practical pedagogical practices, via our observations, experiences, and studies that have been used within the power engineering education. Another goal is to build the awareness of a variety teaching ideas and techniques that a lecturer may consider in light of their current teaching styles and personalities. The problems arising in power engineering education are ranging from complex mathematics and physical concepts, steady-state or time-domain simulations, to lack of adequate phenomenon or solution visualization, being desirable to have access to a computing environment capable of handling such a wide issues, to transform education activities into ones focusing on specific problem aspects rather than extensive learning advanced programming skills or numerical techniques.

Symbolic computational tools or computer algebra systems (CAS), with extensive applications in science or engineering, are designed to obtain explicit problem symbolic solutions ${ }^{1-3}$. A multitude of computational algorithms, tools and techniques are integrated into them to help with the solution, modeling, simulation, editing or visualization. Moreover, software packages, initially designed to numerically solve and visualize mathematical problems are now including 
symbolic capabilities, transforming them into symbolic-assisted numeric computational tools. In the area of power systems, pioneer work was performed in the late 80's at the University of Wisconsin-Madison in the application and use of symbolic computing or symbolic-assisted numeric computational tools in power engineering education ${ }^{3-7}$. There are four CAS features with potential for changing and improving engineering education ${ }^{1-9}$ :

1. Active student learning involvement;

2. Experimentation and simulation as a means of understanding concepts;

3. Solution visualization; and

4. Solving real-world problems.

These themes have continued as the principle set of arguments for CAS using, in the teaching engineering disciplines, particularly in extended uses of CAS-based instruction into laboratories, via simulation, visualization and modeling tools. Traditionally, educators are using dedicated software packages to demonstrate the practical theory applications. These are efficient and suitable to solve a large variety of engineering problems. The students learn programming language and its capabilities to use them for homeworks or projects. There are such computerbased tools available for teaching electrical machines, power system analysis, power electronics or other engineering topics. However, they are specific and confined to dedicated objectives, often lacking the interactivity, and become obsolete once the operating environment is changed ${ }^{5-}$

${ }^{8}$. When dedicated programs are purchased, the buyers receive operation manuals, while the source codes are not usually available and often too complicated to understand. A drawback to use of dedicated programs, as learning tools is that they are requiring intensive training and efforts to develop the links between the software's built in-functions and course materials. These tools are often expensive and have limited capabilities as educational tools, especially, when the educator intends to demonstrate the simple applications. However, the dedicated software could be used for senior or graduate level, where students want to learn industrial problem solving techniques. We are briefly exploring some issues associated with the use of CAS in power engineering education, feedback and inputs from other instructors are highly valuated.

\subsection{Power Engineering Education Issues and Challenges}

The size and complexity of power system or electrical machine problems have questioned the use of dedicated software packages in education. Extensive use of software programs in power industry started few decades ago are playing an indispensable role in power system planning, operation, analysis, modeling, and training. A further growth is anticipated in the development of such software tools in near future with the development of smart grids. Nevertheless power engineering educators waited relatively a long time to respond favorably and to use computing tools as teaching aids. Moreover, several reports suggested that university educational programs in power engineering were far from being healthy ${ }^{2-9}$. However, attempts are made to reenergize power engineering education by incorporating symbolic computation as teaching aid, a natural development since size and complexity of power system problems are making computers essential tools for power system analysis, operation, design or training ${ }^{1-5}$.

The students of electrical power engineering study generation, transmission, distribution and the efficient use of electrical energy, as well as the major power system components, such as: generators, transformers, transmission lines, or control and protection devices. They are using advanced calculus, circuit or signal analysis to solve, model or analyze system components and 
problems. Solved problems are complex and students have not only to understand the topic but also to consider the material influence and device properties is also equally important. CAS can detach slaving away the mathematics, to study problems in their complexity and better understand the concepts ${ }^{3-14}$. Visual information has its indispensable role in understanding the complex power system analysis, operation and characteristics. Features of symbolic computation programs, graphic CAS capabilities are critical and essential here, encouraging students to think visually about power system functions and characteristics. Because of the ease and rapidity with which one can do these numerical computations, symbolic computation programs make feasible to incorporate real-life examples into power engineering curriculum.

Complex problem solution visualization can reinforce the principles learned in classroom, being even greater if the students are exposed to important but ancillary information normally not strictly needed to solve the electric power system problems, such as the appearance of the actual apparatus (size, weight, cost, manufacturer of the apparatus) or the context in which the analysis methods are used. Topics that are normally referred but not fully included in power engineering courses due to time constraints and lack of adequate tools. The pedagogical value of complementing the traditionally presentation of power system or electrical machine analysis and modeling with animated, graphical, easy-to-use computer interface is considerable. Graphical representation of the effect of changing system parameters, topology, boundary and initial conditions provides a dramatic reinforcement to the analytical system knowledge acquired in the classroom. Good engineering education reaches beyond textbook delivery, clever presentation of relevant equations, homework assignments or utilization of innovative grading procedures. It can influence and favorably change student intellectual, analytical, innovative and integrative abilities and motivations, helping to develop critical thinking on issues underlying a problem or solutions. It is in this spirit that many engineering educators have felt the value of using computational tools to complement analytical procedures, enhance subject interests and boost student concept understanding ${ }^{6-14}$. This is even truer for power engineering education, relying on abstract concepts and complex mathematics, often obscuring the intuitive concept understanding, and requiring long learning time from the students to conceptualize underlying physical principles. CAS can provide the needed fresh air to boost power engineering education, revitalize students' interests and significantly enhancing the principle understanding.

\section{Symbolic Computation Packages}

Computations with symbolic data items, a significant part of scientific computation methods, differ from the widely used numerical methods through the way that computation is handled and exactness. CAS major benefit of is that it replaces an often tedious pencil and paper approach for generating symbolic solutions. User is only required to define the problem in a software understandable format while the computer performs the actual calculations. Being user-friendly, it allows students to concentrate on concept understanding of the analyzed problem instead of searching for the methods to solve its mathematics. The student can focus on the engineering work of design and problem solving, while the computational speed allows large number of solutions, providing them with practice in design optimization. CAS provides solutions involving advanced calculus, linear and vector algebra or signal analysis. Hence, they can be applied to solve problems in most of the engineering fields, providing a flexible medium for program developers to assess the numerical behavior of nonlinear models. Symbolic computational 
techniques can be readily used to improve model development and testing. These tools have various advantages, but also some disadvantages, depending on the application environment, being particularly useful for educational and research purposes.

CAS systems, such as MATHCAD, Maple or Mathematica are powerful tools for performing calculations in a power engineering courses. The equations to be solved are written in a format similar to their appearance in textbooks providing an immediate visual feedback, so students have better understanding of the material presented. When using a CAS in class or for preparing homeworks, student focus shifted from learning mathematical methods to concept learning. Several CAS applications to the various engineering and science disciplines have been reported in the literature over the years ${ }^{3,6-12}$. CAS applications in power engineering are very useful for modeling, simulation, the automatic generation of Jacobians and Hessians, code testing, power flow and stability problems. The aim is to obtain explicit symbolic solutions and to gain in the understanding of system concepts. CAS main uses include simplification of expressions, computation of symbolic derivatives and integrals, or elimination and substitution of variables. However, symbolic software packages are regarded as fairly limited in solving large sets of nonlinear algebraic equations and nonlinear optimization problems. CAS core is a powerful symbolically assisted computation engine that, together with user-friendly editor, permits a nearly direct transition from mathematical expressions to simulations. Once the equations are understood, the environment is performing all necessary simulation aspects, allowing a high degree of flexibility. Expressing a problem statement in natural form or experimenting with a problem as much as necessary makes CAS very attractive for power engineering education. The equations are entered in an intuitive manner with few rules, can be arranged in any desired order, and text can be intermixed at any time. There are another two most important CAS features. The screen equation appearance matches as closely the textbook equation forms, a very helpful feature, whiles the built-in measurement units, is particularly helpful because the students can concentrate on the meaning of what they learn and know the transformation is done properly.

For power engineering optimization problems the computation range from unconstrained to inequality constrained problems. CAS, for example enables handling different mathematical forms of optimization problems, including automatic recognition of the problem type and problem setting in the form of Lagrangian and Kuhn-Tucker optimality conditions ${ }^{2-7}$. Among many, CAS environments offer the following features: symbolic and numeric equation linearization, simplification, symbolic elimination, handling complex numbers and equations, repetitive solutions, or signal analysis. Each problem can be saved as a lesson file for later use. This is the point where the instructors can benefit from the environment by preparing problems to be solved and storing them as the lessons with solving instructions (all CAS have capabilities to differentiate between ordinary text and equations) ${ }^{2-7,12}$. CAS also include: quick and correct way of deriving exact analytical results, short calculations, flexibility, easy algorithm developments, beautiful post-calculation numerical solutions and graphics, or easy error detection. CAS is an effective teaching and research tool. Symbolic languages often produce shorter and more reliable programs than conventional programming languages. Important steps in using symbolic programs are: 1) THINK first with care and know concepts, 2) TEST cases, from easier ones to difficult and complex cases, 3) USE different systems simultaneously, avoiding long expressions, and 4) EMPLOY extensively the CAS graphical capabilities. 
The development of symbolic manipulation codes, such as MACSYMA, MATHEMATICA, MAPLE, and AXIOM marks a breakthrough to the computing and programming techniques having enhanced capabilities of working with symbols and enabling to perform lengthy and complicated analytical operations with higher speed and reliability. The most popular among CAS are Mathematica ${ }^{\mathrm{TM}}$, Mathcad, Maple ${ }^{\mathrm{TM}}$ and MATLAB. Other systems include AXIOM $^{\mathrm{TM}}$, MACSYMA, MuPAD ${ }^{\mathrm{TM}}$ or REDUCE ${ }^{\mathrm{TM}}$. All systems can be used for solving complex problems, but they differ in comfort and complexity and each has a different look and feel. Maple, for example has some features, such as handling of partial differential equations that others do not have and, from the author's personal point of view, the user interface is easier to handle than that of the competitors. MACSYMA was the first CAS, developed in 1959 at Massachusetts Institute of Technology, MATHEMATICA by SMP, Interference Corp., in 1970 and upgraded by Wolfram Research Inc., in 1988. MAPLE is a product of Waterloo Maple Software Inc., 1980, developed originally at University of Waterloo, Canada to assist science and engineering students to undertake mathematical operations on a computer. Although, Maple has become a major commercial product, its devotion to educational mission remains steadfast, setting a standard. Freely available CAS software applications are ready through internet including comprehensive courses in traditional areas of mathematics, science and engineering. Other symbolic packages include: REDUCE (RAND Corporation, 1970), DERIVE (muMATH, 1970; and Soft Warehouse Inc., 1988), AXIOM (Scratchpad II, IBM Research Center, 1970; NAG Library, 1991), Mathcad, Mathscribe, Cayley, MaCaulay, PowerMath, Scolar, etc. All these symbolic computation packages are quite unexpansive at least in the academic license versions.

\section{Symbolic Computation Applications in Power Engineering Education}

Symbolically-assisted numeric computation refers to the use of symbolic computation not as an end in itself, but rather as a means of assisting in the numeric solution of a problem. Major CAS applications to power systems and electric machines simulation, analysis and modeling, include the determination of electromechanical oscillation modes in power systems, time-domain electromagnetic transient problems, transient analysis of electrical machines, or the solution of a problem involving interacting nonlinearities. Symbolic-assisted computation has now become part of the mainstream programs used for the analysis, modeling and simulation of power systems. A good example of how symbolic computation can be used in the development of commercial-grade software for power system analysis is that Jacobians and Hessians can be readily and reliably obtained symbolically for a variety of power system models to then integrate them into any numeric computational package. From the educational side, the computational techniques are complementary for several reasons ${ }^{6-12}$. First, concept understanding may be greatly enhanced through the use of computational tools, while in some problems it is better to use symbolic techniques rather than numerical evaluations. In some cases solutions of this kind are very useful for the interpretation of results. Symbolic computations are applicable for lots of mathematical manipulations particularly differentiation, integration, linear equations, function expansions, differential equations, curve analysis, or tensor algebra. Either CAS tools have been around for a number of years or widely used in some areas, many people have been put off using such tools for cost or time reasons. However, CAS environment are now available on a wide range of machines, with similar or identical platform interfaces, while inexpensive site licenses are available. User interfaces are reasonably friendly, with good help systems. Graphics facilities are excellent, and graphs can be saved in a variety of formats suitable for imbedding in papers or reports. Most systems enable the user to output single equations or whole sessions in $\mathrm{TeX}$ or 
LaTeX which can be pasted directly into a paper. There is also a large user community, and help is available from user groups. There also are a number of well-written manuals and books, with Maple and Mathematica scoring highly in this respect. Last but not least, any CAS can be easily linked to numerical packages and other programing languages. Most CAS can be put to use immediately, and the user can be obtaining useful results within short time. However, it takes longer time of frequent use to be familiar with all the CAS potential features and techniques.

Our CAS assisted applications were included in our energy conversion, electric machines and power engineering courses. First course is the Electric Machines and Energy Conversion, designed to introduce the structure, analysis and principles of electromechanical systems (magnetic circuits, transformers, DC and AC rotating electrical machines) and application. This course discusses major classes of static and rotating electrical machines, characteristics, tests, equivalent circuits and performances, tools and techniques used for electrical machines operation and control. The second course, Introduction to Power Engineering is designed to provide the students with an overall introduction to the generation, distribution and use of electrical energy in three-phase balanced systems. Topics include: fundamentals of energy-handling electric circuits, transmission lines, linear and rotating electric machinery, power system representations, use of lumped parameter electro-mechanics to understand power systems, models of synchronous, induction, and DC machinery, the power system interconnections, transmission and distribution system structure, transmission line modeling for steady-state analysis, network calculations, power flow analysis, and controlling bus voltages and power flows. The first set of developed worksheets refer to power system components and elements, such as: transformers, generators, electric motors, transmission lines, real and reactive power control, fault analysis, transient stability analysis, power quality issues, as well as general discussions of computer applications to power systems, network modelling and calculations. To extend and take the full advantage of the capabilities and features of CAS environments in power engineering courses, a set of programs and applications were developed or are underway to be developed in Maple or Mathcad to analyze and model: magnetic circuits, electric machines, transmission lines, balanced and unbalanced three-phase circuits, power flow analysis problems, voltage and current controllers. Among the CAS applications and worksheets developed and tested include:

1. Asynchronous induction machine with slip-ring rotor;

2. DC machine losses models;

3. Magnetic circuits

4. Induction machine losses model;

5. Permanent magnet synchronous machine;

6. Three-phase transformers $Y-Y$ windings;

7. Single-phase transformers;

8. Space phasor components

9. DC series machines and shunt machines;

10. Voltage-frequency controller;

11. Symmetrical components.

12. Symmetrical faults; line-to-line, line-to-ground, and line-to-neutral.

Other courses are the Power Electronics and Power System Analysis and Design. Both are upper level undergraduate courses offered in combination with similar graduate courses, having 
different requirements. We are focus here on the design and implementation of advanced power system analysis problems. For example, practical analysis of power system stability requires consideration of bifurcation phenomena, requiring numerical procedures using higher order derivative information than conventional analytical methods ${ }^{7-9}$. The analysis of complex bifurcation problems can be carried out on very simple power systems, using CAS for education purpose. In our application, bifurcation analysis is the study of behavioral variations within families of nonlinear systems. Usually, in power system studies, these families are explicitly defined as parameter dependent sets of differential and algebraic equations. For this reason, symbolic computation could be useful to generate them. Symbolic constructions are also required for two distinct purposes: model building and analysis. Modeling, for example a power electronics converter necessitates deriving many expressions, describing the converter operation or testing alternative design solutions. Symbolic computation may assist the students in design and analysis of several classes and types of power electronics converters.

\section{Pedagogical Considerations}

Power engineering students study generation, transport, distribution and the efficient use of electrical energy, relatively wide branch of engineering, requiring extensive use of mathematics as an essential part of it. Solved problems are complex and difficult to handle. CAS can help to study power engineering problems in their complexity. Visual information has in indispensable role, here. Another feature of symbolic computation programs commonly used is their ability to do infinite precision numerical computations. Such computations are frequently used to illustrate points about functions and limiting processes. Similarly one can demonstrate facts about derivatives by computing difference quotients at various points close to the point in question. Because of the ease and rapidity with which one can do these numerical computations, symbolic computation programs make it feasible to incorporate such examples into the curriculum.

Developed worksheets are structured hierarchical, consisting of an introduction and one or more subdivisions or topics. The content of introductory section may differ according to the purpose and the specific worksheet application. For supplementary class notes, theoretical presentation of the subject is appropriate, adequate references are given to keep the introduction shorter. If the worksheet is providing answers for homeworks, then the problems to be solved are stated in the introduction. To merely refer to a problem number in a textbook is not sufficient. In addition, the homework that students submit must have the problem accurately stated. To save students the time a worksheet containing only the problem may be provided. The main part of the worksheet may be divided into two sections. The first section presents the theoretical analysis, being kept in symbolic format where possible. The second section is reserved for numerical calculations, presenting and discussing the results, and checking the correctness of the answers.

Pedagogic considerations make important to keep calculations symbolic as long as possible. For instance, symbolic expressions can show the relationship between components in a system, circuit or device, whereas a numerical value gives no indication of any such dependencies. Symbolic results of a calculation, for example a transfer function, are therefore important for students to better judge and evaluate the validity of the analysis. Students may have tendency to do numerical calculations and to resist doing symbolic manipulations. If numerical answers to a problem are required, as is often the case, they must be obtained and checked at the end. A 
worksheet written by the instructor therefore needs to stress the importance of doing the symbolic analysis before obtaining the numeric results. As teachers, we must require the same of our students. Another important point is that the student must always be required to analyze the correctness of the obtained results. Worksheets need to encourage students to do further work on a subject. Any worksheet must contain questions and problems for students to solve. I have used two ways of posing questions in a worksheet. First, the worksheet has short questions helping the students focus on important problem aspects that may result in modifying and adding it. Second, questions are requiring students to elaborate further on problem understanding, or requiring them to do additional calculations on similar problems or other problem aspects.

\section{Student Response and Lessons Learned}

There is no truer statement than the following: If a CAS is to be experienced in a lab setting, then precise and well-crafted exercises and projects are essential. Students having unpleasant CAS experiences are not likely to enjoy the extra effort needed to master it. This desideratum is difficult to achieve. Almost any variety of instructional modes is suitable for inclusion of a CAS. Faculty preparation time and enthusiasms are important contributors to the success. Overall, the initial use of CAS in a course requires a lot class preparation than the traditional way of teaching. Usually, for the experienced instructor, new material development requires shorter preparation time for each hour of classroom time than for new instructors. It is also helpful, if teaching assistants are well versed in the CAS, to the level of being able to develop large programs and to fully use CAS capabilities. However, keep in mind that CAS systems are only tools and are no replacing subject matters. The software allows a student to concentrate on the final solutions by spending minimum time on lengthy mathematical computations and/or tedious programming and numerical computations. The benefits offered by these systems are significant and should be exploited in the undergraduate technology curriculum towards enhancing students' knowledge and for demonstration of the "what-if" analysis which is commonly performed in engineering practice. The surveys for power engineering courses, were extensively used CAS as teaching aid are rather similar. The questionnaire included a question on the students' expectations of the use of these packages in the course, to later compare with their actual observations and use of these tools. Most of the students viewed favorable and gave positive feedbacks to the CAS inclusion. However, most of the critics refer to to extend the CAS presentation and materials to the beginning of the course and the addition of extra CAS tutorials over the semester.

\section{Conclusions and Future Work}

We have discussed the role symbolic computation plays in teaching power engineering courses. The presented general-purpose symbolically assisted numeric computation environment can be used directly as an educational and research tool. CAS is a standard a tool for students and engineers for doing applied mathematics, performing design calculations and solving complex engineering problems. However, CAS systems are not able to perform miracles, since all the symbolic calculations could in principle be done by hand. CAS can open up a field of computations that are often practically impossible to carry out manually. Similar applications to other areas of engineering are possible since no matter which engineering problem is solved; one always ends up with a similar formal problem description. Symbolic computational techniques and tools have various advantages and disadvantages, depending on the application environment, 
being particularly useful for applied science educational and research purposes. It is important to highlight the fact that as the computing power has significantly increased in recent years the types of applications of symbolic computation have also increased considerably. However, computational burden is still an issue that limits the direct use of symbolic computational techniques and tools in commercial-grade applications for power system studies. Features of symbolic and numerical software packages were briefly discussed. The test results improved and student interest increased significantly since the introduction of the interactive problem solving in a computer equipped classroom. Most students like the use of the computers in the classroom and they are able to solve advanced problems that cannot be solved by hand calculations. Complaints were received from weak students. The students use the computers during the openbook final exam and midterm tests. The advantages of the proposed method are:

1. The students write the equations in the familiar textbook format.

2. The problems are solved interactively, increasing student interest.

3. The students analyze the results and draw conclusions, which enhance their learning.

4. These programs are frequently used by industry, another significant advantage to use them.

5. No need to learn programming techniques or dedicated input-output type computer programs.

\section{References}

1. P. R. Wellin. A letter from the Editor. Mathematica in Education, 1991, Vol. 1(1), pp. 1.

2. T. Schramm, Computer Algebra Systems in Engineering Education, Global J. of Eng. Educ., 1998, Vol. 2(2), pp. 187-1994.

3. M. N. Pavlovic, Symbolic computation in structural engineering, Computers \& Structures, 2003, Vol. 81(22), pp. 2121-2136.

4. C. A. Canizares, and Z. T. Faur, Advantages and disadvantages of using various computer tools in electrical engineering courses, IEEE Transactions on Education, 1997, Vol. 40(3), pp. 166 - 171, DOI: 10.1109/13.618025.

5. R.G. Belu R.G. and A.C. Belu - Teaching Electrical Engineering Using Symbolic Computation, 2005 Annual ASEE Conference Proceedings, June 12-15, Portland, Oregon, 2005. (CD Proc.).

6. C. Buteau, N. Marshall, D. Jarvis, Z. Lavicza, Integrating Computer Algebra Systems in Post-Secondary Mathematics Education: Preliminary Results of a Literature Review, International Journal for Technology in Mathematics Education, 2010, Vol. 17(2), pp. 57-68.

7. R.G. Belu R.G., Teaching Mechanics with Maple, 2011 ASEEE Conference \& Exposition, June 26 - 29, Vancouver, BC, Canada (CD Proceedings).

8. M. H. Nehrir, F. Fatehi, and V. Gerez, Computer modeling for enhancing instructions of electric machinery, IEEE Trans. Educ., 1995, Vol. 38, pp. 166-170, May 1995.

9. F. L. Alvarado and C. A. Canizares. and, Symbolically-Assisted Power System Simulation, International Journal of Electrical Power \& Energy Systems, 1996, Vol. 18(7), pp. 405-408.

10. G. G. Karady, Life Fellow, and K. A. Nigim, Improve Learning Efficiency by Using General Purpose Mathematics Software in Power Engineering, IEEE Transactions on Power Systems, 2003, Vol. 18(3), pp. 979-985.

11. M. Glavic, I. Dzafic, and S. Tesnjak, A General-Purpose Symbolically Assisted Numeric Computation Environment as a Support in Power Engineering Education, IEEE Trans. on Power Systems, 2005, Vol. 20, pp. 3-12.

12. C. Domnisoru, Using MATHCAD in Teaching Power Engineering, IEEE Transactions on Education, 2005, Vol. 48(1), pp. $157-161$.

13. A. Lamari, Modelling and simulation of electrical machines on the basis of experimental frequency response characteristics, Journal of Computer Engineering Research, 2010, Vol. 1(1), pp. 7-13.

14. A.M. Dąbrowski, S.A. Mitkowski, A. Porębska \& E. Kurgan, Formulating structural matrices of equations of electric circuits containing dependent sources, with the use of the Maple program, $1^{\text {st }}$ World Conference on Technology and Engineering Education 2010 WIETE, Kraków, Poland, 14-17 September 2010, pp. 66-70. 